Published in IEEE International Symposium on Biomedical Imaging (ISBI) 2009

\title{
3D EIGENFUNCTION EXPANSION OF SPARSELY SAMPLED 2D CORTICAL DATA
}

\author{
Moo K. Chung ${ }^{1,3}$, Yu-Chien $W u^{3}$, Andrew L. Alexander ${ }^{2,3}$ \\ ${ }^{1}$ Department of Biostatistics and Medical Informatics \\ ${ }^{2}$ Department of Medical Physics \\ ${ }^{3}$ Waisman Laboratory for Brain Imaging and Behavior \\ University of Wisconsin, Madison \\ mkchung@wisc.edu
}

\begin{abstract}
Various cortical measures such as cortical thickness are routinely computed along the vertices of cortical surface meshes. These metrics are used in surface-based morphometric studies. If one wishes to compare the surface-based morphometric studies to 3D volume-based studies at a voxel level, $3 \mathrm{D}$ interpolation of the sparsely sampled $2 \mathrm{D}$ cortical data is needed. In this paper, we have developed a new computational framework for explicitly representing sparsely sampled cortical data as a linear combination of eigenfunctions of the 3D Laplacian. The eigenfunctions are expressed as the product of spherical Bessel functions and spherical harmonics. The coefficients of the expansion are estimated in the least squares fashion iteratively by breaking the problem into smaller subproblems to reduce a computational bottleneck.
\end{abstract}

\section{INTRODUCTION}

Cortical surfaces have been characterized by various geometric measures such as cortical thickness [5] [7], curvatures [3] [12] and area elements [6]. These measures are computed along the vertices of cortical surface meshes. After surface normalization, these measures are feed into statistical analysis pipelines. Surface specific analysis tend to sensitize surface specific tissue change and has been used frequently in quantifying the amount and the shape of gray matter change. The limitation of surface based approaches is the additional computational burden of segmenting gray matters accurately and obtaining cortical surfaces meshes.

On the other hand, the volume-based morphometric techniques such as the deformation-based morphometry [2] [6] or voxel-based morphometry [1] do not require the additional step of obtaining cortical surface meshes. If one tries to compare or combine both surface- and volume-based measures, one has to transform the measurements into a common space. Since the voxel space is more densely defined than mesh vertices, it is easier to warp volume measures to a surface. In [5], the computation intensive nearest neighbor search algorithm [9] on an optimized k-D tree is used to compute the distance map and warp volume measures to a cortical surface mesh. If one wishes to warp surface measures such as cortical thickness to the 3D volume space, one has to interpolate voxels that the mesh vertices do not pass through.

In this paper, we present a new explicit functional representation technique to address the problem of resampling sparsely sampled cortical data to a densely defined volume space. The cortical data is represented as the linear combination of basis functions, which are the eigenfunctions of the 3D Laplacian. The eigenfunctions are the product of spherical harmonics and spherical Bessel functions. Our approach should offer more unified modeling flexibility than widely used radial basis approaches [4] [8] since each basis has the identical mathematical form. On the other hand, the radial basis method represent data as the linear combination of low degree polynomials and radically symmetric functions.

The eigenfunction expansion of cortical data is not a computationally easy problem due to the large number of mesh vertices upward of 700000 . If one tries the traditional least squares estimation [10] [14], one encounters a serious computational bottleneck of solving 700000 linear equations simultaneously. Using the recently developed iterative residual fitting algorithm [5], we reduce the computational burden to solving few equations at a time. Our framework is very general that it can be directly applicable to constructing the probability density function that describes water diffusion from multiple shell data without much modification in the framework [16] [17].

\section{EIGENFUNCTION EXPANSION}

Suppose the Cartesian coordinates $\left(p_{1}, p_{2}, p_{3}\right)$ are given by the spherical coordinates $(r, \theta, \varphi)$ as

$$
\left(p_{1}, p_{2}, p_{3}\right)=(r \sin \theta \cos \varphi, r \sin \theta \sin \varphi, r \cos \theta),
$$

where $(\theta, \varphi) \in[0, \pi] \otimes[0,2 \pi)$. Define the spherical Laplacian on the unit sphere $S^{2}$ as

$$
\Delta_{S^{2}}=\frac{1}{\sin \theta} \frac{\partial}{\partial \theta}\left(\sin \theta \frac{\partial}{\partial \theta}\right)+\frac{1}{\sin ^{2} \theta} \frac{\partial^{2}}{\partial \varphi^{2}} .
$$



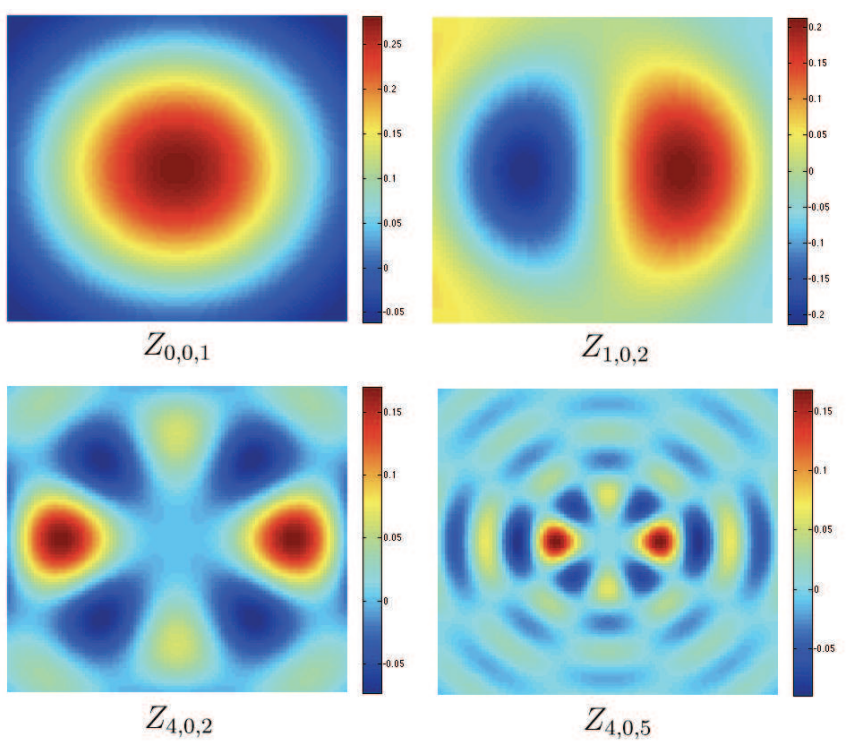

Fig. 1. Basis functions $Z_{l m n}$ are visualized in the cube $[-1,1]^{3}$. The images are the cross sections at $p_{2}=0$ The expansion is only valid within the ball of radius 1 . The indices of $Z_{l m n}$ corresponds to the spherical harmonic $Y_{l m}$ and the $n$-th root of the spherical Bessel function $S_{l}$. The index $n$ basically relates to the scale of the pattern $Y_{l m}$ in the radial direction.

The Laplacian in the solid ball $\mathcal{M}$ of radius 1 is then defined as

$$
\Delta_{\mathcal{M}}=\frac{\partial^{2}}{\partial r^{2}}+\frac{2}{r} \frac{\partial}{\partial r}+\frac{1}{r^{2}} \Delta_{S^{2}}
$$

using the spherical coordinates $(r, \theta, \varphi)$. Consider the eigenvalue problem

$$
\Delta_{\mathcal{M}} f+\lambda f=0
$$

in the solid ball of radius 1 . We may assume the additional Dirichlet boundary condition

$$
f(r=1, \theta, \varphi)=0
$$

Substituting the separable solution of the form

$$
f(r, \theta, \varphi)=g(r) h(\theta, \varphi)
$$

in (2), we obtain

$$
r^{2} \frac{g^{\prime \prime}}{g}+2 r \frac{g^{\prime}}{g}+r^{2} \lambda=-\frac{\Delta_{S^{2}} h}{h}=\mu
$$

for some constant $\mu$.

We first solve for the second equation

$$
\Delta_{S^{2}} h+\mu h=0
$$

The solutions to (4) are the spherical harmonics $Y_{l m}$, where $l$ and $m$ are called the degree and the order respectively. The

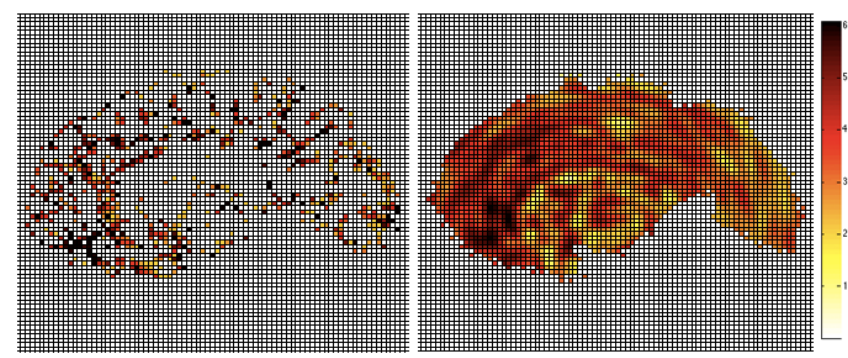

Fig. 2. Left: the cortical thickness defined on mesh vertices are rounded to the closest voxel. Right: Eigenfunction expansion with degree 22 and 22 roots. Only the masked brain region is shown. The representation can fill out voxels where cortical thickness is not defined.

explicit form for spherical harmonics is given in [5]. The eigenvalues are $\mu_{l m}=l(l+1)$ for $l=0,1,2, \cdots$. The first equation can be written as

$$
r^{2} g^{\prime \prime}+2 r g^{\prime}+\left[r^{2} \lambda-l(l+1)\right] g=0 .
$$

If we define a new variable $g=r^{-1 / 2} G$, we can transform the equation to

$$
r^{2} G^{\prime \prime}+r G^{\prime}+\left[r^{2} \lambda-\left(l+\frac{1}{2}\right)^{2}\right] G=0 .
$$

This is the scaled version of the Bessel equation and the only bounded solution at the origin is given in terms of the Bessel function of the first kind as $G(r)=J_{l+\frac{1}{2}}(\sqrt{\lambda} r)$. The solution to $(5)$ is given by

$$
g(r)=r^{-1 / 2} J_{l+\frac{1}{2}}(\sqrt{\lambda} r) \propto S_{l}(\sqrt{\lambda} r),
$$

where $J_{l+\frac{1}{2}}$ is the Bessel function of the first. The solution is proportional to the spherical Bessel function $S_{l}$ defined as

$$
S_{l}(x)=\sqrt{\frac{\pi}{2 x}} J_{l+1 / 2}(x) .
$$

The first term of the spherical Bessel function is

$$
S_{0}(x)=\frac{\sin x}{x} .
$$

Other terms are obtained recursively from

$$
S_{l+1}(x)=-S_{l}^{\prime}(x)+\frac{l}{x} S_{l}(x) .
$$

Few other terms are

$$
\begin{gathered}
S_{1}(x)=-\frac{\cos x}{x}+\frac{\sin x}{x^{2}} \\
S_{2}(x)=\frac{\sin x}{x}-\frac{3 \cos x}{x^{2}}+\frac{3 \sin x}{x^{3}} .
\end{gathered}
$$



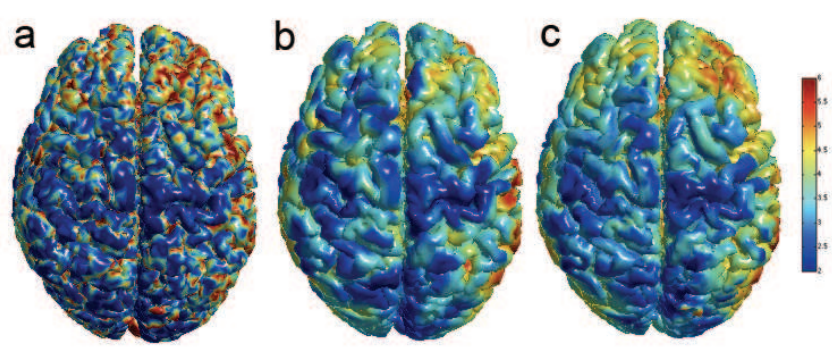

Fig. 3. (a) Cortical thickness usually ranges from 2 to $6 \mathrm{~mm}$. (b) Eigenfunction expansion with degree $k=22$ and $j=5$ number of roots. (c) Eigenfunction expansion with $k=10$ and $j=22$.

In the computer implementation of the spherical Bessel function, one may need to define $S_{l}(0)$. However, the built-in spherical Bessel functions in most computer programs such as MATLAB cause the singularity at $x=0$. Hence we need to define $S_{l}(0)$ explicitly. Using the l'Hospital's rule, we have $S_{0}(0)=1$ and $S_{1}(0)=0$. Then using the l'Hospital's rule iteratively, we have

$$
\lim _{x \rightarrow 0} S_{l+1}(x)=-\lim _{x \rightarrow 0} S_{l}^{\prime}(x)+l \lim _{x \rightarrow 0} S_{l}^{\prime}(x)=0 \text { for } l \geq 1 .
$$

Since the solution should satisfy the boundary condition (3), we should have $S_{l}(\sqrt{\lambda})=0$. We order the roots of the spherical Bessel function as

$$
0<\sqrt{\lambda_{l, 1}}<\sqrt{\lambda_{l, 2}}<\sqrt{\lambda_{l, 3}}<\cdots .
$$

For the 0 -th degree, the roots are trivially given as $\sqrt{\lambda_{0, n}}=$ $n \pi$. All higher roots are numerically estimated.

Multiplying the spherical Bessel functions and the spherical harmonics together, the eigenfunctions to (2) are then given by

$$
Z_{l m n}(r, \theta, \varphi)=S_{l}\left(\sqrt{\lambda_{l n}} r\right) Y_{l m}(\theta, \varphi) .
$$

Figure 1 shows the representative basis sampled in the cube $[-1,1]^{3}$ at the cross section $p_{2}=0$. These eigenfunctions from a basis within a solid sphere of radius 1 . Then any function $f \in L^{2}(\mathcal{M})$, the space of square integrable functions, can be expanded as

$$
f(r, \theta, \varphi) \approx \sum_{l=0}^{k} \sum_{m=-l}^{l} \sum_{n=1}^{j} \beta_{l m n} Z_{l m n}(r, \theta, \varphi) .
$$

The expansion is truncated at the degree $l=k$ and with $n=j$ roots.

\section{ITERATIVE RESIDUAL FITTING ALGORITHM}

Previously the coefficients of spherical harmonic series expansion have been estimated using the least squares method by solving the system of linear equations [5] [10] [14]. For a cortical surface mesh with $N$ vertices, we need to simultaneously solve $N$ linear equations and, in turn, invert an $N \times N$ matrix. For cortical surface meshes, $N$ can easily reach up more than 700000 and it will not fit most computer memories. To address this computational bottleneck, we have developed the iterative residual fitting algorithm [5] that divide the extremely large linear problem into manageable small subset of linear problems.

Let $p_{i}=\left(r_{i}, \theta_{i}, \varphi_{i}\right)$ be the mesh vertices where the cortical measurements $f$ are given. We vectorize the measurement as

$$
\mathbf{f}=\left(f\left(p_{1}\right), \cdots, f\left(p_{N}\right)\right) .
$$

Let $\mathbf{Z}_{l,, n}$ be the $N \times(2 l+1)$ submatrix of basis given by

$$
\mathbf{Z}_{l,, n}=\left[\begin{array}{ccc}
Z_{l,-l, n}\left(p_{1}\right) & \cdots & Z_{l, l, n}\left(p_{1}\right) \\
\vdots & \ddots & \vdots \\
Z_{l,-l, n}\left(p_{N}\right) & \cdots & Z_{l, l, n}\left(p_{N}\right)
\end{array}\right] .
$$

Denote the matrix of all basis corresponding to the $l$-th degree as $\mathbf{Z}_{l}=\left[\mathbf{Z}_{l,,, 1}, \cdots, \mathbf{Z}_{l,, j}\right]$. Define the vector of coefficients corresponding to $\mathbf{Z}_{l}$ as

$$
\beta_{l}=\left(\beta_{l,-l, 1}, \cdots, \beta_{l, l, j}\right)^{\prime} .
$$

Then we iteratively estimate the coefficients of low degrees to high degrees using the iterative algorithm.

Algorithm 1 Iterative Residual Fitting.

1. $l \leftarrow 0$.

2. $\mathbf{r} \leftarrow \mathbf{f}$.

3. $\beta_{0} \leftarrow\left(\mathbf{Z}_{0} \mathbf{Z}_{0}\right)^{-1} \mathbf{Z}_{0}^{\prime} \mathbf{f}$.

4. $l \leftarrow l+1$.

5. $\mathbf{r} \leftarrow \mathbf{r}-\mathbf{Z}_{l-1} \beta_{l-1}$.

6. $\beta_{l} \leftarrow\left(\mathbf{Z}_{l} \mathbf{Z}_{l}\right)^{-1} \mathbf{Z}_{l}^{\prime} \mathbf{r}$.

7. If $l \leq k$, go to Step 4 .

\section{3D RESAMPLING OF 2D CORTICAL DATA}

Among various cortical measures, we have used the cortical thickness to demonstrate the proposed method. High resolution magnetic resonance images were obtained using a 3Tesla GE SIGNA scanner. The collected images went through intensity nonuniformity correction [15]. A supervised neural network classifier was used for tissue segmentation [11]. Subsequently a deformable surface algorithm was used to obtain both the inner and the outer cortical surfaces that bound gray matter [13]. The cortical thickness is then defined as the 


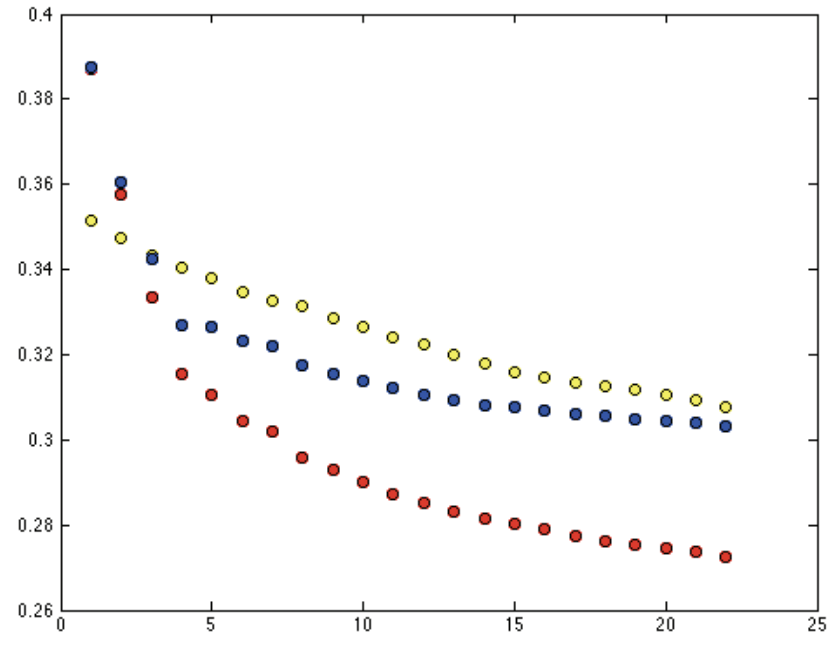

Fig. 4. Relative error plot of eigenfunction expansion for various number of degrees and roots. Yellow dots are the errors for varying number of degrees for the fixed number of roots $j=5$. Red (blue) dots are the errors for varying number of roots at the fixed degree $k=20$ (10). As expected, the error plots show the increasing the number of degrees and roots increases the accuracy.

distance between the two surfaces along the vertices of the cortical mesh (Figure 3).

Since the brain surfaces can not be contained in a ball of radius 1 , we have scaled the mesh coordinates to be contained in the ball. Then we have performed the eigenfunction expansion. As the degree and the number of roots increases, the expansion should be able to represent more detailed cortical pattern. Taking the given cortical thickness as the ground truth, we have computed the average relative error between the ground truth and the representation (Figure 4). Increasing the number of roots and degrees decreases the discrepancy between the representation and the ground truth. Since there are so many empty voxels where cortical thickness is not defined (Figure 2), the effect of the eigenfunction expansion is more like low-pass filtering.

\section{ACKNOWLEDGMENT}

The authors wish to thank Ameer Hosseinbor of University of Wisconsin at Madison for discussion on the spherical Bessel functions. The research is funded in part by NIH MH62015.

\section{REFERENCES}

[1] J. Ashburner and K. Friston. Voxel-based morphometry - the methods. NeuroImage, 11:805-821, 2000.

[2] J. Ashburner, C. Hutton, R. S. J. Frackowiak, I. Johnsrude, C. Price, and K. J. Friston. Identifying global anatomical differ- ences: Deformation-based morphometry. Human Brain Mapping, 6:348-357, 1998.

[3] A. Cachia, J.-F. Mangin, D. Riviere, F. Kherif, N. Boddaert, A. Andrade, D. Papadopoulos-Orfanos, J.-B. Poline, I. Bloch, M. Zilbovicius, P. Sonigo, F. Brunelle, and J. . Regis. A primal sketch of the cortex mean curvature: a morphogenesis based approach to study the variability of the folding patterns. IEEE Transactions on Medical Imaging, 22:754-765, 2003.

[4] J.C. Carr, W.R. Fright, and R.K. Beatson. Surface interpolation with radial basis functions for medical imaging. IEEE Transactions on Medical Imaging, 16:96-107, 1997.

[5] M.K. Chung, L. Shen Dalton, K.M., A.C. Evans, and R.J. Davidson. Weighted fourier representation and its application to quantifying the amount of gray matter. IEEE Transactions on Medical Imaging, 26:566-581, 2007.

[6] M.K. Chung, K.J. Worsley, S. Robbins, T. Paus, J. Taylor, J.N. Giedd, J.L. Rapoport, and A.C. Evans. Deformation-based surface morphometry applied to gray matter deformation. Neurolmage, 18:198-213, 2003.

[7] B. Fischl and A.M. Dale. Measuring the thickness of the human cerebral cortex from magnetic resonance images. PNAS, 97:11050-11055, 2000.

[8] M. Fornefett, K. Rohr, and H.S. Stiehl. Elastic registration of medical images using radial basis functions with compact support. In IEEE Computer Society Conference on Computer Vision and Pattern Recognition, pages 402-407, 1999.

[9] J.H. Friedman, J.L. Bentley, and R.A. Finkel. An algorithm for finding best matches in logarithmic expected time. ACM transactions on mathematics software, 3:209-226, 1997.

[10] G. Gerig, M. Styner, D. Jones, D. Weinberger, and J. Lieberman. Shape analysis of brain ventricles using spharm. In $M M$ BIA, pages 171-178, 2001.

[11] K. Kollakian. Performance analysis of automatic techniques for tissue classification in magnetic resonance images of the human brain. Technical Report Master's thesis, Concordia University, Montreal, Quebec, Canada, 1996.

[12] E. Luders, Thompson, P.M., K.L. Narr, A.W. Toga, L. Jancke, and C. Gaser. A curvature-based approach to estimate local gyrification on the cortical surface. NeuroImage, 29:12241230, 2006.

[13] J.D. MacDonald, N. Kabani, D. Avis, and A.C. Evans. Automated 3-D extraction of inner and outer surfaces of cerebral cortex from mri. NeuroImage, 12:340-356, 2000.

[14] L. Shen, J. Ford, F. Makedon, and A. Saykin. surface-based approach for classification of $3 \mathrm{~d}$ neuroanatomical structures. Intelligent Data Analysis, 8:519-542, 2004.

[15] J.G. Sled, A.P. Zijdenbos, and A.C. Evans. A nonparametric method for automatic correction of intensity nonuniformity in mri data. IEEE Transactions on Medical Imaging, 17:87-97, 1988.

[16] D.S. Tuch. Q-ball imaging. Magnetic Resonance in Medicine, 52:1358-1372, 2004.

[17] Y.-C. Wu and A.L. Alexander. Hybrid diffusion imaging. Neurolmage, 36:617-629, 2007. 